\title{
CONCORDIA ENTRE EL LUGAR DEL GRADO Y LA CIUDAD DE ZARAGOZA REALIZADA EN 19 DE NOVIEMBRE DE 1463
}

$352(091)$

\author{
por \\ Enrique Orduña Rebollo
}

El Ayuntamiento del Grado conserva cuidadosamente una serie de documentos en su Archivo que constituyen su patrimonio histórico más apreciado. El referido Ayuntamiento, a pesar de las dificultades comunes a todas las Corporaciones para conservar sus documentos, ha hecho esfuerzos en tal sentido y tiene la favorable cualidad de disponer de personas siempre interesadas en el tema, tanto el Alcalde como el Secretario que actualmente dispone la Corporación.

Del conjunto de documentos conservados en su Archivo municipal, uno de ellos es al que se refiere el presente estudio y transcripción realizada a instancias de la Corporación a través del Centro de Documentación del Instituto de Estudios de Administración Local, y clyas características y texto expondremos seguidamente.

El documento por nosotros estudiado es la «Confirmación por el rey FeLIPE IV, de la concordia hecha entre el lugar del Grado en este Reyno de Aragón y la ciudad de Zaragoza sobre la unión de aquel lugar con esta ciudad». Este documento real está promulgado en Zaragoza el día 20 de noviembre del año 1642, signado por el notario real, Gaspar DE TORRES, y el escribano mayor del reino, Bernardo SANZ de CUENCA.

Se trata de un documento en pergamino de nueve folios que corresponden a 18 páginas de $35 \mathrm{~cm}$. de largo por 24 de ancho. De la página 3 a la 18 forman un cuadernillo, estando cosido el pri- 
mer folio (páginas 1 y 2) con grapas al cuadernillo y parece ser está cortada la que correspondería al folio 10 (páginas 19 y 20), aunque no compromete la integridad del documento.

Presenta una marca de doblez al centro en el sentido longitudinal, no muy acusada.

En buen estado de conservación general. El folio 1 está cortado en el margen longitudinal exterior y los números 4 y 7 en el margen inferior, impidiendo la lectura de una palabra en la página 1, línea 3, no quedando afectado el resto del texto. En el folio 7 , página 13, también se encuentran afectadas unas palabras de la última línea. Los cortes parecen haber sido realizados por medio de instrumentos afilados, pues no se observa desgarradura violenta.

Salvo en el folio primero, en los demás se encuentran unas huellas de mordedura de roedor en la parte superior del margen lateral exterior que no afectan a la lectura ni al texto. No hay señales de que el documento pueda haber estado sometido a la acción de la humedad.

En cada folio, en la parte superior izquierda existe un sello circular en tinta morada del Ayuntamiento del Grado.

Los actos públicos descritos en el documento se realizan ante el notario Antón DE LA Cuesta en el año 1463.

Son capítulos, hechos y concordados entre el Jurado, Capítulo y Concejo y Concejo y Universidad de Zaragoza, de una parte, y el honorable Antonio DE LA EsPLuga, de la otra.

En ellos se acuerdan, entre otras cosas, que haga venta Antonio DE LA EsPluga a dichos Jurados, de los referidos castillo y lugar del Grado con sus términos y con los hombres, jurisdicción, etc. Que se haga la concordia entre la ciudad de Zaragoza y el lugar del Grado. Que los Jurados de Zaragoza y sus sucesores en el cargo no puedan enajenar ninguna parte o totalidad de los dichos castillo, lugar y jurisdicción, etc., del Grado, y si tal caso sucediera pierda ipso facto todos sus derechos la ciudad de Zaragoza. Asimismo se determinaba que no podían maltratar de hambre ni matar de sed a los de dicho castillo y lugar, ni usar contra ellos de absoluta potestad. Los vecinos del pueblo, entre otras cosas, podrían hacer uso del término, montes, tierras, aguas y leñas lo mismo que los vecinos de los barrios de la ciudad de Zaragoza. $\mathrm{Y}$ así sucesivamente.

El acto público tuvo lugar en las denominadas «Casas del Puen- 
te», de la ciudad de Zaragoza, el 19 de noviembre de 1463. Dos días más tarde, en Barbastro y ante el mismo notario, comparecen don Antonio de la Espluga y su esposa, doña Leonor de la PUerTa, los cuales aceptan todos y cada uno de los capítulos de la venta. El precio estipulado es el de veinte mil sueldos, que afirma haber recibido el señor DE LA ESPLUGA; la venta queda refrendada por el citado Antón DE LA CUESTA, notario público de Zaragoza.

Hemos consultado, asimismo, el primitivo documento, que también se conserva en el Archivo del Ayuntamiento del Grado, que fue elevado en 1552 a la confirmación real, que tuvo lugar, como ya hemos indicado, en 1642, y del emanado en esta fecha la transcripción del texto es como sigue:

NOS PHILIPPUS DEI GRATIA REX Castallae Aragonum Legioni vitriurq Siciliae, / Hierusallem, Portugallia, Hungaria, Dalmatiae, Croatiae, Nauarra, Granata, / Valentia, Gallaetiae, Maionicarum, Hispalis, Sartinia, Corduba, Corsiuae, Murtia, / Giennis, Algarby, Algezira, Gibraltaris, Insulae Canariae, nec non Indiarum/Orientalium, et Occidentalium, Insulae et terra firmae, Maris Occeani, Archi / dux Austriae, Dux Bugundiae, Brabantiae, Mediolani, Atheniensi et Neopatriae / Comes Abspurgy, Flandriae, Tirolis, Barcinonae, Rosillonis et Ceritaniae / Munchio Cristani, et Comes Goceani. Partium convectiones et Contractus inter/vassallos mii, et primati, magnum Regis confirmatio nen in mementum validitu / tem suscipiunt. Idem cum proparte Loci sec Opidi del Grado m hoc Aragonum / Regno nobis fuere deductum, et expositium interipsum, Juratos et Concilium Civita / tis Caesaraugustae quamdam circa conditiones et iurisdictionem cumquibus dictum / loci del Grado ditta ciutati se adhaesit aggregauit, et forem se gentulit praece / dente Episcopi Merdensis Laudatione puisse pactum translactionem et concor / diam eius tenor seguitur sub his verbis. In Dei Nomine Amen. Nouerint / Uniuersi quod Anno a Natty. Millem. quingentesmo. quinquagesmo. secundo / die vero quae computabatur decima quarta mensis Juny apud civitatem aeltae Rgeni / Aragonum, et in Aula superiori Diputationis eiusdem regni obi Curiae Domini Jus / titiae Aragonum solita est celebrari ac hora celebrationis eiusdem, et coram multi Magco. / et Prouido regni obi Curiae Domini Jus / titiae Aragonum solita et celebrari ac hora celebrationis eiusdem, et coram multi Magco. / et Prouido viro Domino' Antonio de Vera Jurisperito, Locumtenente multum Magnifici / et Circunspecti viri Domini D. Ferrary de Lanu za militis serenissimi Domini / Nostri Regis Conciliary, ac Justitiae Aragonum in Judicio pro tribunali sedente / et causas publice, et more solito audiente, et celebrante, ubi multa turba aduocatorum procuratorum, et aliarum quamplurimarum existebat, Comparuit personaliter Constitutus / discretus vir Joannes Della Causidieus Caesaragustae hauitator, et procurator et eo / nomine magnifi- 
corum Dominos, Juratorum, Conciliy, et Universitatis, ac singularum / personarum vicinorum, et habitatiorum dictae civitatis caesaraugustae, qui / quodam exhibuit Instrument um publicum, Capitulorum, et concordie in hitae, fac / tae e concordatae inter dictos juratos, Concilium, et Concilium et Universitattm dictae / civitatis Caesaraugustae ex vna, et Magnificos Antonium de la Espluga, Dominun/Castri, et Loci del Grado, et Dominan Leonorem de la puerta, conjuges domiciliatos / in civitate Barbastri, et intimationes et Laudationes eiusdem partibus exaltera / Pargameno exaratum non viciatum, Chancellatum, nec ni aliqua ipsius exalteraspectum, / hed omni propsus vitio, et suspitione carens, exceptis albis in eodem instrumto. obmisi claudere propter ipsius vetustatem et Lacerationem pro ut ipsius facie prima / apparebat thenoris sequentis. In Dei nomine. Amen. Sea atodos manfiesto / que llamado siquiere cidado Concello de Jurados, Concelleros, prohombre de la / por Miguel de / Belchite Corredor publico dela dha ciudad mediante Martín de Lodossa, e Pedro / de Ripaldo trompetas, et con sonamiento de trompas por las plaças, e lugares acostum/brados de la dita ciudad, segun que del dito llamamiento el dito Corregidor fizo rela/ción, presentes los testimonios dins escritos ami Anton de la Cuesta notario, diuso nombrado, e ajustado el dito Concello en las casas Comunes vulgarmte llamadas Las Casas del Puente de la dita Ciudad, en do et según que otras vegadas portales e semblantes / actos, como lo dius escritos en dito Concello se es acostumbrado plegar e agustar / en tl cual fueron presentes los que se siguen Don Luis de la Cavallería, Don Jaime Sanchez / Don Domingo Sebastián, Don Juan de Fatas, jurados, Don Luis de Palomar, Don Pedro Torrellas / Don Alphonso Francés, Don Juan Marco, Don Miguel de Fuertes, Don Jaime Oliván / Tristán Bartholomeu Ferrer, Juan de Aguas notts. García / Cariñena, García Salvatierra, Berenguer de Chipre, Andreu de Pertussa, Miguel / de la Torre, Pedro Cruillas Concelleros, Bartholome de Lastaneta, Pero de Altarribas, / Juan Zaidi, Gil de Luesma, Domingo Prouales, Joan del Río, Gaspar de Alberuela / Gabriel Bercossa, Gil de Barbuesta, Nicolau Ginero, Bartholome Ibañez / y Bartholome Gascon García Moya, Miguel Nauarro, Luis de Palomas, Pero Cruillas, Juan Albiol, Pero Lopez de Aynzon, Gonzaluo de Santa María, Sancho / del Gele, Juan Rosel, Miguel Juan Mayor, Juan de Salabert, Juan Daynas / Juan Torralba, Juan de Torres, Salbador Serra, Pedro Monya, Jayme Ferrer / Anton Noble, Miguel Bello, Pero Martinez de Alforez, Garcia Ferrer, Do/mingo de Astruga, Jaime Blasco, Juan de Soria, Juan de Balconchans, Mar/tin de la Zarda, Pero Colado, Anton Manzan, Juan Gallart, Agustín de Arbus / Luis Salbador, Martin Rubio, Anton de Monçon, Juan de Monçon, Anton Castro, / Juan de Rello, Juan de Soria, Jaime Menente, Anton Carro, Juan de Cumto, Mar/tín Viello, Ximeno de Soria, Lorent Rosell, Miguel Laraz, Paulo Ferrer, / Pasqual de Luerma, Bartholomeu Roca, Juan Nauarra, Juan Dorunyo, Pas/qual Gallego, Anton Nauarro, Miguel de Fuertes, Martin de Lodossa, Anton / Plaça, Guillem Balsa, Pero Martínez de la Rambla, Martín Vicent, Do/mingo Agustin, Lázaro de Boran, Domingo... de Cuesta, mayor / Miguel de Arbanies, Anton de Ar- 
guis, Thomas Bon, Simón Monient, Gal/lart Donchiant, Antrih Bages, Garcia Moya, Juan de Rodera, Anton Ballari / Anton Bernard, Juan Barba, Pedro Asmtiano, Anton de Piniella / Juan de Briuiesca, Gabriel Moliner, Juan de la Ram, Tristán de la Porta / Pedro de Luna, Pero Marco, Saluador Barrachina, Alphonso de Castro, Juan de Taraçona, / Anton de Pertussa, García de Moros, Franch. Roper, Misser Paulo / Villanovadellas, Juan de Mazallon, Roldan Guarnimenttero, Nicolas Çapatero / Juan Domingo, Luis de Esplugas menor, Pedro Sastre, Log Caquero, Pedro Balbán, / Francisco de Montesor, Matheo de Erica, Bartholomeu de Cortes, Ferran Caualler / Juan Gram, Ramon Vera y Salvador dela Mayson, Ciudadanos, vecinos de la dita Cidad. / Concellantes y Concello facientes, en el qual concello, por el dito Don Luis de la Caualleria / jurado fue messo en caso quel Lugar del Grado, se daua adaquesta Ciudad / en aquesta manera, quel dito lugar se sauia quitado de Pedro de Bardaxi, e por q / ellos se pudiessen dar aquien quisiessen sauian feyto fazer Lavenditton de la / dita compra a Antoni Laspluga Ciudadano dela ciudad de Barbastro en el dito / Antonio de la Espluga... por veyte mil sueldos, de los quales la Ciudad non de Sauia de pagar les, et / sobre aquesto se Sauian feyto e ordenado, ciertos capítulos entre la dita ciudat / de una parte, et el dito Antonio Laspluga de la otra, Los quales capitulos hauian / sido vistos y reconocidos por Aduogados de la dita Ciudat, et por los Jurados / Capitol y Concello, epor otros muytos Ciudadanos de aquella, a los quales $\mathrm{Ha}$ / bia parecido seyer buenos, et que la Ciudat, si quiere concello deaquella et / el dito Antoni Laspluga deuer otorgar, lohar et aprobar aquellos, a fin / que llohados, aprouados et confirmados, et dito Antoni Laspluga ficiesse / vendiccon a la dita ciudat siguiese Capitol, o Concello, et Concello de aquella / del dito lugar, et el dito Concello era llamado por aquesta razon, et assi que / viessen que se devia fasser, que deliuerado por el dito Concello y los ditos capitales / feytos, e concordados entre la dita ciudat de una parte, et el dito An / toni despluga de la otra seleyessen en el dito Concello deliuerasse que se deuia / fazer, Los quales Capítulos fuessen leydos publicamte. / en el dito Concello por mi dito notario, Los quales son del tenor siguiente. Capitulos feltos e con / cordados entre los Magníficos senores Jurados, Capitol, e Concello, e Concello / e Universidad de la Ciudat de Caragoça de la una parte, et el honorable / Antoni de la espluga, ciudadano de la Ciudat de Barbastro dela parte otra / e net sobre el infrascrito. Primeramte. es concordado entre el dito Antoni de / La espluga señor que es de los Castillo e lugar del Grado faga válida vendición / a los ditos Jurados, Capitol e Concello, e concello e Universidad de la dita Ciudaz / de Caragoza de los ditos castiello, e lugar del Grado con sus terminos, e con los / hombres e mulleres en aquellos habitantes, et hauitadores, e con la Jurisdiccion / civil y criminal, alta e vaxa, mero e mixto imperio, e al exercicio deaquellos / pertenecientes, excepto los dreitos, e otras cosas de la parte diuso eximidos / reseruados e reseruadas por precio de vint mil sueldos, los quales confessa hauer / recivido La qual vendición los ditos Jurados, Capitol e Concello e Concello e / Uniuersidad 
de la dita ciudaz de Caragoça acceptaran con los pactos, condiciones, / reseruaciones e retencciones infrascritas es a saber et sesiguen- Que los / ditos Jurados, Capitol e Concello, e Uniuersidade de la dita ciudaz / de Caragoça, qui ahora son, e por tiempo seran en tiempo alguno, ni por qualquier, / tiempo, quanto quiere poco por qualquier necesidad quanto quiere extrema, ni / en manera alguna no pueden dar, vender, permutar, transportar, aliertar, empenyar, / atributar, ni en uso, usofructo, $o$, en otra manera atorgar en todo, o en parte quanto / quiee mínima los ditos Castillo et Lugar, términos, Jurisdiccion, e cient sueldos / infrascriptos ni las otras cosas infrascriptas, o, de alguna deellas, ni aquellos / ni aquellas, o, alguna deellas separar á todos tiempos, ni por algún tiempo quanto / quiere mínimos de la dita Ciudat de Caragoça, antes sia ad acto alguno de / donaccion, vendiccion, permutaccion, transportaccion, alienaccion, Impignoraccion, / atributacion, $o$, concession en usofructo o vsufructo, directamente, o indirectamente per / uendran que no valga, ni tenga las ditas vendiccion, donaccion et todas las alie / nacciones sussodichas, e qualquiera deelas sean ipso facto et foro, cassos e cassas / irritas e nullas, et en tal caso la Ciudadt Caragoça ipso facto et forme pierda / el precio de los ditos vint mil sueldos sobreditos, et ipso facto et foro la dita / vendiccion et qualquier dominio, propiedad, posession, e los cient sueldos / infrascriptos de la dita ciuddat de Caragoça, et qualquiera que en virtud de la dita vendiccion / se habia subseguido sea resoluida et resoluida en todo lo sobredito et quialquiera deellos ad / quiridos ipso facto et foro a los Jurados, Conctllo e Universidad del dito Castillo / et lugar del Grado sequedan liberament fines restituccion alguna del precio de los / ditos vint mil sueldos sobre ditos dar al señor Rey, Iglesia, orden et qualquiere Ciudat / Villa, o, Lugar, de la señoría del Rey de Aragón, et agora por la ora siaperdido / el dito precio de los vint mil sueldos ipso facto et foro et la vendicción nulla, et / sean liberos et francos de qualquiere sagrament, é homenaje de lealtad ala / dita Ciudad habran prestado los Jurados, Concello e Uniuersidad et singulares / de dito Castillo e Lugar del Grado, et quien los ditos Jurados, Capitol y Concello / et Concello e Uniuersidad de la dita Ciudat de Caragoça que los ditos Castiello, e Lu / gar, terminos jurisdiccion e cient sueldos et otras cosas sobredichas sea assi vnidas / e vnidas incorporadas e incorporados a la dita ciudat de Caragoça, como si por / fuero et acto de Corte fuessen a daquella unidos, e unidas incorporados e incorpora / das, assi que en tiempo alguno, ni por qualquiere tiempo, quanto quiere poco de / aquello nose puedan separar, et sean tenidos, et obligados á deffender que por el / señor Rey, ni por sus successores, ni por otra persona alguna no sean separados de la / dita Ciudat de Caragoça et adeffenderlos en los Privilegios, liuertades, franquesas e inmunidades de la dita ciudad. = ITEM quieren los ditos Jurados, Concello / e Uniuersidad de la dita.Ciudad de Caragoça de la una par, et el ditho Antoni / dela Espluga de la otra, e con tal pacto, condicion, reservacion, se fara la dita / vendiccion que en aquella no sean comprehensos, e comprensas las premicias, alcaualas / treudos, emolumentos de fornos, tornos, 
molinos, et otras qualesquiera rentas que agora / son, o, por tiempo sean, ni el dreito del Patronado del dito Antoni como señor / del dicho castiello e lugar del del Grado, et a la señoria del dito Castiello /, e del Lugar de la presentaccion del Retor en la Retoria dela Iglesia del señor San / saluador del dito lugar pertenecientes antes de la dita vendiccion sean exiuidos / e exibidas, e exclusos e exclusas, e que en las ditas primicias, treudos, emolumentos / de tornos, fornos, molinos, rentas presentes, aduenideras, et dreyto de patronado so / bre ditos e sobre ditas, Los ditos urados, Concello e Uniuersidad de la dita Ciudad / de Caragoça no hayan dreyto alguno, antes las ditas premicias treudos, emolumentos, / de tornos, fornos, molinos, rentas, y otaras coasas sobreditas, presentes et aduenideras / sean validamente para todos tiempos perpetuo de los Jurados, Concello / e Uniuersidad del dito Castiello e Lugar del Grado, qui ahora son, o, por tiempo / serán en los quales el dito Antoni dela Espluga señor sobredito en aquella / millor manera, via et forma que mas validamente puede los da et transferace / quanto al Patronado de consentimiento del Vispe de Leryda diocesis del / dito lugar, et quien las ditas partes, que quantas vezes de aqui adelante / para todos tiempos vacara la dita Retoria, los Jurados, Concello e Uniuersidad / del dito Castiello e lugar del Grado, e los singulares de aquel, o, la mayor / parte de dito Concello hombres de dito castillo e Lugar, presentes, e adue / nideros ad imperpetuum puedan por su procurador, como Patrones de la dita / Retoria presentar á quien bien visto les sera, e que los Jurados, Concello, / e Uniuersidad de la dita ciudat de Caragoça, directamente, ni indirecta no les puedan / meter en todas las cosas sobreditas, qualquiera decellas e contraste / alguno. ITEM quierem las ditas partes, e con tal pacto, e condiccion se fara / la dita vendiccion que los ditos Jurados, Concello e Uniuersidad del Grado que / agora son, e por tiempo seran touidos en cada un año dar e pagar de compartimiento / a la dita ciudad de Caragoça e al mayordombre de aquella en el dia e fiesta / de cabodaño en cascunaño, cient sueldos dineros lagueses, et que dicho Com / partimiento, peyta, morauedi, pontaje, ni cargo alguno Rey al, personal/. e patrimonial, ordinario, o extraordinario, o, otor alguno por la dita Ciudat Imposada, / o, Imposadero, no sean tenidos ni obligados, ni obligados los ditos Jurados, Concello / e uniuersidad, e singulares e términos presentes e aduenideros del dito Castiello / e Lugar del Grado, de los sobreditos cargos, et de cualquiere de ellos et de repa / ración, o, construccion de muros puentts, carreras e otros cargos, por la dita / Ciudad imposados e imposaderos, sean liberos e francos exceptos los ditos cient / sueldos de compartimiento. ITEM es concordado entre las dos partes y los / ditos Jurados, Concellos e Uniuersidad del dito Castiello e Lugar del Grado que, da facer cotos, estatutos e paramientos, con que empeio siean confrimados por / Los Jurados, Capitol, y Concello de la dita Ciudad de Caragoça, o, por la mayor / parte. ITEM es concordado entre las ditas partes, que los ditos Jurados, Concello, / e Uniuersidad del dito Castiello e Lugar presentes e advenideros / o, la mayor parte puedan en cada un año liuremente servir concelleros, 
Jura / dos, Mucasafe, vedaleros, monteros, corredores, carniceros, paniceros, et / otros qualesquiere oficiales menores de jurados en el dia de cabodaño, e de alli ade- / lant qualquiera día, a los quales, et a qualquierede ellos los Jurados del dito Castiello / e Lugar tomen las Juras. Los quales e qualquiere deello hayan todo aquel / poder, que de fuero, uso, e costumbre del Reyno de Aragon pueden e deuen sauer / et que no hayan de ser confirmados por la dita ciudad de Caragoça ni por los / Jurados, Concello e Uniuersidad de aquella, ni en las ditas electiones, et / toma de jura, empacho alguno no les puedan poner. ITEM es concordado / entre las ditas partes que los ditos Jurados, e Concello del dito lugar presentes /, e aduenideros, o, la mayor parte de ellas puedan é ayan en cada un año en el mes / de deziembre elegir tres personas, o, por su letra, o, en otra manera, Intimar / a los Jurados de Caragoça que la hora seian, e aquel de los ditos tres que por los / ditos jurados de Caragoça, o, por la mayor parte de ellos sera electo, sea sauido por / Justicia en el dito Castiello e Lugar, ejerze ordinario para el año siguiente / de alli adelante fasta en tanto que el del año siguiente pueda vsar, el qual / Justicia electo segun dito es por los Jurados de Caragoça haya toda aquella / Jurisdiccion que ejerza ordinario de fuero puede, et deue sauer, el qual Justicia / sobredito en caso de absencia, o, enfermedad, o, justo impedimento, pueda crear / Lugartiniente, el qual haya toda aquella Jurisdiccion que habria el dito Justizia / del Judicco del qual Justicia, o, Lugartenientes sobreditos los hauitantes, / o habitadores en el dito Castiello e Lugar no puedan seyer sacador, sino por / appellacion segunt de usso se contiene. ITEM es concordado entre las ditas / partes, e con tal pacto se haze la dita vendiccion, que los Jurados, Concelleros, / e singulares, e Concello del dito Lugar del Grado, presentes e advenideros, et / qualquiere de ellos se pudan appellar del Justicia, o, de su Lugartiniente del dito / Lugar del Grado, e de las sentencias, e pronunciacciones de ellos, et de qualesquiere / deellos a los Jurados, o, á la mayor parte deellos de la dita Ciudad, o, a los / Jurados, Capitol, e Concello de aquella al qual mas placera alappellaccion /. ITEM es concordado entre las ditas partes, e, con tal pacto se face la dita / vendiccion que los Jurados, e, Ciudadano infranscrito de la dita Ciudad en / su caso, et los ditos Capitol y Concello en el suyo, e los ditos Jurados, e / Lugartinientes de Justicia en su caso, et qualquiere deellos en las causas, assi / ciuiles, como criminales que conoceran tocantes al dito castiello, e / Lugar, Jurados, Concello, Uniuersidad, e singulares, de aquel presentes / e aduenideros hayan de proceder segun los estatutos, cotos, e / paramientos de dito castiello e lugar, et segun los fueros, liuertades, vsos e costumbres / del Reyno de Aragon, et quieren, e con tal pacto e / condicion se faze la dita vendiccion, que los ditos Jurados, Concello e Uniuersidad / e Capitol e Concello de la dita Ciudad de Caragoça presentes e aduenideros no ç puedan maltratar ni defambre, ni de sed matarlos, los Jurados, Concello, e / Uniuersidad, e singulares del ditto Castiello, e Lugar presentes, e aduenideros, ni alguno / deellos, ni contra aquellos vsar de absoluta potestad, antes, adaquel fuero, e / 
observancia de maltratar et de fambre et sed matar expressamente renuncian. / et que hayan a se usar los ditos statutos, cotos, e paramientos, fueros, usos, / costumbres del Reyno de Aragon, o no puedan otramente vsar procedimiento / excepto aquel fuero, e obseruancia sobredita, al qual, o, los expresamente / Renuntiando segun dito es /. ITEM es condicion entre las ditas partes, e con / tal pacto e condicion, se faze la dita vendiccion, que los ditos Jurados, Concello, / e Uniuersidad, e singulares del dito Castiello, e Lugar, presentes, e adue / nideros hayan todo aquel uso en los terminos, montes, hieruas aguas / lenyas, aljez, piedras, caças que los Jurados, Concello e Uniuersidad / e singulares de los varrios antiguos de la dita Ciudad de Caragoça / e en los terminos de los varrios de la dita ciudad. / ITEM es concor / dado entre las ditas partes, que los ditos Jurados, Concello e Uniuersidad / e singulares de la dita Ciudad, quanto fazer lo pueden acullen a los / ditos Jurados, concello, e Uniuersidad e singulares personas presentes, e / aduenideras, e terminos presentes e aduenideros del dito Castiello, e / Lugar, e a todos sus bienes sauidos y por sauer e aplican por todo su poder / et quanto fazer lo pueden á todos, e qualesquiere privilegios, liuertades / immunidades et franquesas que la dita Ciudad de Caragoça, et los hauitantes en / aquella et los varrios antiguos de aquella, han ni hauer pueden, et de aqui auiat / sabran los quales privilegios, Liuertades et franquesas, e immunidades quie / ren los ditos Jurados, Concello e Uniuersidad de Caragoça seyer sauidos / aqui por chalendados, nominados, expecificados, assi como expresamente / todos, e qualesquiere deellos fuessen chalendados, nominados y especificados, el / qual acullimiento e applicamiento de los ditos privilegios, liuertades e / franquesas en todo, quanto quiere nonimá Los Jurados, Concello, / e Uniuersidad de la dita Ciudad, reuocar, no puedan et si / lo facian, que ipso foro et facto sia resoluida et nulla, et inualida la / dicha vendiccion e los actos por virtud de aquella subseguidos segunt lar / gamente es dito en el Capitol primero. ITEM es concordado entre las dichas partes quesi por vandosidades, $o$, necesidad otra el Concello, del dito Casti / ello e Lugar, o, la mayor parte de aquel deliuerara seyer neccessario / que un Jurado, o, Ciudadano de la dita Ciudat elaya al dito Castiello / e Lugar, que en tal caso la dita Ciudat Loy de aya de enviar á expensas / del dito Castiello, e lugar, con plena Jurisdiccion e pottestad, empero sino / hiran los Jurados todos, o, part, o, Ciudadano, á suplicación del dito Lu / gar segunt dito es, que en tal caso los Jurados, Concello e Singulares / del dito Castiello e Lugar, presentes et aduenideros no sean tenidos de / pagar las expensas ni adaquello, et que de aquellos, o, de quelquiere / dellos que ivan e exhimir en el dito Castiello e Lugar segun es dito / se puedan apellar a los Jurados solos, o, a los Jurados, Capitol, y / Concello dela dita Ciudad de Caragoça a quien mas el appellante / querra. ITEM es concordado entre las ditas partes, con tal pacto i condicion se / fara la dita vendiccion, que si Los Justicia, Jurados e Officiales del dito Lugar / del Grado sobreditos, e quelquiere deellos faran contra los statutos del dito Cas / tiello Lugar, o, contra los Fueros, vsos e cos- 
tumbres del Reyno de Aragon / al Concello del dito castiello e Lugar, $o$, a los singulares de aquel o cualquiere / deellos, que quedan seyer auisados de todo lo sobredito por aquel de / qui sera intereses de lant de los Jurados de la dita Ciudat, o, la mayor part / de aquellos, o, de los Jurados, Capitol y Concello de la dita Ciudad delant / qual mas querra el acusant, e Leydos visto e reconocidos los Capitoles / por los ditos Jurados Concelleros, e por todo el dito Concello o Uniuersidad / dela dita ciudad, Concellantes, e Concello facientes, Concordablemente que delibe / rado por la dita Ciudat si quiere Concello, e Uniuersidad de aquella deuia / tomar e tomasse el dito Lugar del Grado con los ditos Capitoles, pactos, condicciones / retencciones, e Limitacciones en aquellos contenidas de susso largamente / insertos, et que compre el dito Castiello, e Lugar con los ditos Capitoles, pactos, / condicciones, retenciones, e Limitacciones en aquellos contenidos largamente / desusso insertos. Por precio de los ditos vint mil sueldos los qualeshatorgue / hauer reciuido el dito Antoni Laespluga, los quales Capitoles et cada uno / deellos, e cada uno deellos cantenidas los ditos Jurados et Concelleros, e todo / el sobredito Concello, e Uniuersidad de la dita Ciudad Concellantes, / e Concello facientes concordablemente de sus ciertas sciencias del dieyto de / la dita Ciudad bien, y plenamente certificados en todo, et por todas las cosas, / dixeron que pues nada no constaua como de ñelto no constaua a la dita ç Ciudat de Carago/a segunt mi notario insfrascrito consta y lo hacian / firmauan y aprouauan, ractificauan e emologauan e confirmauant segune quees / feyto loaron, firmaron, aprouaron, ratificaron y emologaron et segunt / queen los ditos Capitoles et cadauno deellos desusso se contiene, et / prometieron e se obligaron tener, seruir y cumplir los ditos Capitoles, y cada / uno de ellos et todas e cada vnas cosas en aquellos contenidas et contra aquellos / ni aquellas no venir ni permitir seyer venido en algun tiempo por algun caso / dieyto, manera o razon, et a estto tener, seruir, obligar y cumplir obligaron todos los / bienes y rentas de los Jurados, Capitol y Concello, Concello e Uniuersidad / de la dita Ciudat de Caragoça, nobles e sedientes, sauidos y por sauer / en todo Lugar. Feyto que aquesto en las ditas casas del Puent de la / dita Ciudad de Caragoça à diez y nueve de noviembre Anno á Nattivita / Domini Millessimo Cuadrigentessimo sexagesimo tertio. De Testimonios fueron / a lo sobredito presentes Castan de la Cambra et Martin de Vera, hauitantes / en la dita Ciudat de Caragoça apres de losobredito dia que secontauan / a veyntitres del mes sobredito de Nouiembre anyo de sussodito de / mil quatrocientos xixenta y tres, dentro en la Ciudat de Barbastro en / presencia de mi dito notario y de los testimonios infrascritos parecieron / los muy honorables Don Antoni de La espluga, ciudadano de la dita / ciudat de Barbastro, señor del Castiello e lugar del Grado, e Doña Leo / nor de la Puerta muller del dito Antoni de la Espluga, Los quales dixeron / que attendido y considerado que entre los ditos Jurados Capitol e Concello, / Concello e Uniuersidad de la dita Ciudat de Caragoça de una parte / et el dito Antoni de la Espluga de la otra eran concordados, pac / tados e fechos los Capitoles de la parte de sus- 
su insertos sobre la venta / (las últimas palabras mutiladas por corte del folio) del dito Castiello e Lugar del Grado querienteś lo pactado concordado, e / fecho deducir a deuido effecto. Por tanto los ditos Don Antoni de la Espluga / y Doña Eleonor dela Puerta Conjuges vistos, leydos e entendidis los ditos / Capitoles de susso insertos, certificado plenariamente de su dieyto en todo e por /todas cosas dixeron que Loauan aprobauan, ratificauan, emologauan e afir- / mauan, segun que de feyto loaron, firmaron, aprouaron, ratificaron, / et emologaron los ditos Capitoles de la parte de sussu insertos, et cada uno de ellos / et todas e cada unas cosas en aquellos et cada uno de ellos prometió y se obligó / tener, seruir e cumplir los ditos Capitoles, et cada uno de ellos, et todas, y / cada unas cosas en aquellos contenidas et contra aquellos ni aquellas / ni venir ni permitir seyer venido en algun tiempo, ni por algun caso, dieyto / manera, o razon, e aquesto tener, seruir y cumplir obligaron todos sus / bienes muebles e sedientes sauidos y por sauer en todo lugar. Feyto fue / aquesto en la Ciudat de Barbastro, día, mes et y año supradito, testimonios / fueron a las sobreditas cosas presentes Domingo de Amigo notario, et / Juan de Monclus ciudadanos de la Ciudad de Barbastro. Nos / Antonius Aguelana, Vicarius Generalis Rmi. Domini Cardinalis / Episcopi Merdensis decretorum. Doctor Canonicum Merdensis Suic praeinserti / Capitulationis ac Patroni nostrum imponimus decretum propia que auece / manu propia suscribens dieltanno quo supra, et sigillum imprimi / mandantes. Sig. (sello) no. de mi Anton de Curla notario publico dela / Ciudad de Caragoça, y por autorizacion del Sr. Rey, por los Reynos. de Aragon / y Valencia qui a las sobreditas cosas presente fue et las primeras dos / lineas, chalendarios e nombres de testimonios de mi propia mano / (línea mutilada por corte del folio). escriui, met lo otros seruiri fize et cerre, consta de sobrepuestos en la Lm lineas dosehe / a los de la dita ciudat en la Lxy dose lie, o, et en la Lxy dose lie del quo / quidem instrumento publico capitulationis, et concordia, intimationis, et Laudationis / eiusdem sic perdictum Joannem della Prqm. praedictum de super exhibitio e idem proisi / dixit quod cum dicti eius principalis velmt, et intendant se tam in presenti Regno / Aragonum quam extra in diversis Regnis, et partibus, et tam in Judicio quam / extra iubare, et sibi et dictus eius principalibus videatur usse valde perien lo sum / propter inundationes aquarum, et alia discrimina, que vinire et oriri possent, ideo / idem procurator, petyt suplicauit, et requisiuit praedictum dominim Locrimtyem. / Justitiae Aragonum dictum, et praeinsertum Instrumentum publico in libro, sine / regestro actuum Communium Curiae dictii Domini Justitiae Aragonum registrare / et apponi mandare, eidem quam suam impedere auctoritatem, pariter et decretum / et sigillo, et armis eiusdem curiae muniri, et sigilare mandare, ut de eodem / transumpto tam injudicco, quam extra fides indubitata adlibeatur, et dictus / Dominus Locumts auditis praedictis, visoque dicto Instrumento publico Capitu / -lationis et Concordiae desuper inserto ni sui prima figura non viciato, nee can / cellato nec in aliqua ipsius parte suspecto sed omni prorsus vicco et suspitione / carente, demptis duobus albis quae propter 
Lacerationem ipsus Legi non potuerunt / dicto procurem. instante per notarium curiae seu in Libro sue registro communi / actum praedictae curiae scribi, registrari, inserti et apponi mandauit et de / eodem transumptum auctenticum fieri pussit, et praedictum transumptum / auctorizauit eidemque atribuit dedie, et impendit auctoritatem paritem et decre / tum dughis verbis. Un de Nos Antonius de Vera Jurisperitus Locumts. / multum Mag. ci .et circunspecti vin Domini Ferrary de Lanuça militis se / renissimi Domini Nostri Regis Consiliary, ac Justitiae Aragonum, audita / et nitellecta dicta petitione, perdictum Joannem Della procuratorem prae / fatum facta, visoque dicto originali instrumento publico concordiae, Intimationis /. et Laudationis eiusdem desuper in sui primafigura coram nobis exhibitio non viciato, / nec Cancellato, nec in aliqua eius parte suspecto, sed amni prorsus vitio, et suspitio / ne carente impsumque in dicto libro siui regesto communi actuum dicta curiae Domini / Justitiae Aragonum inseri, registrare et apponi mandamus, et de eodem transumptum / auctenticum freri jubemus ipsumque transumptum auctorizauimus, et eidem nostram / auctoritatem et decretum impendimus, sigilloque dicta curiae Domini Justiciae Aragonum / impendente sigillari mandamus. Volentes et decernentes quod eidem transumpto / impedicio quam extra ad imperpetium fides plenaria adhibeatur tampm. dicto / et supra inserto Instrumento Publico. Qua fuerunt acta in loco, menses, et / anno desuper in principio calendatis, praesentibus ibidem protestibus discretion / viio Michaele de Albacar et Martino Perez notarys Regyo habitatoribus / Civitatis Cesaraugusta. Sig. Finum mei Gasparis de Torres habitato / is Civitatis Caesaraugusta autoritateque Regia per totum Regnum Aragonum / notaryi publici, et Regentis substituti Cuiusdam ex scribanis Curiae Domini / Justitiae Aragonum pro Mag.co. Bernardo Sanz de Cuenca Scriba principali / dictae Curiae, et Scribaniae qui huiusmodi Instrumentum publicum transsumpsi / a suo originali Libro siue regestro aituum Communiumm, et emparamentorum / presentis Curiae, et Scribaniae anni Millessimo quinguntessimo quinquagessimo secundo / ubi continuatum existit manualiena extraxi, et Comprouani in quorum / fidem et testimonium meo solito signo signaui, et cum sigillo communi dictae / Curiae sigillatum juxta Aylum eiusdem. Fuitg proptersa Majestati ntra. / humiliter supplicatum, vt promaiore et frimiore dictae transactionis et Concordiae robore / et firmitate impsum, et omnia et singula mea contenta Laudare, approbare, ratificare / et confirmare, et quattens opus sie de nouo Concedere dignaremur. Nos vero Commo / dum vtilitatem dicti Loci del Grado, nec non Civitati Caesaragustae per / pendentes et considerantes petitioni illorum benigne annuere decrevimus / tenore igitur praesentis de ntra. certa sciencia, Segraq. auctze. deliberar et / consulto praedictan, et praeinsertam transactionem, et concordiam et omnialt singula / ri ea contenta, a prima eius Linea, vsp. ad vltiman juxta ipsius seviem, et tenorem / Laudamus, approbamus, ratificamus et confirmamus, et quattenus opussit de nous / concedimus et elargimum nraeq. huiusmodi Laudationis, approbationis, ratificatio / nis, et confirmationis munimine seu praesidio roboramus 
et validamus volentes, et / expresse deciinentes, quod nostra huiusmodi Laudatio approbatio, ratificatio et Con / firmatio et quattenus opus sit noua concessio fit dictis Loco del Grado et Civitati / Caesaraugustae ac singutaribus earum realis valida atque firma nullumque injudiccio / aut extra sentiat dubitatis objectum, deffectus incommodum aut noxe euius li / bet alterius detrimentum, sed in suo semper robore et firmitate persistat. Sup / plentes, et tollenter ex nostrae Regiae potestatis plenitudine omnes, et quorumque / deffectus, et solemnitatum omnis fiones, siquae velquae inpraemisis interuenient / aut quomodo libet annotata fuerunt insimi Libus fieri requirantur Serenissimo propterea Balthasar Carlos Principi Asturianum, et Gerundae, Duiq. Cala / briae et Montis Albi, fili primogenite nostro Massimo, ac post felices, et Lon / geuos dies nostros in omnibus Regnis, et Dominys nostris (Deo propitio) im / mediato haeredi et Legitimo sucessori intentum apperientes nostrum sub paterna / bendictionis obtentu dicimus, eumque rrogamus, futuro vero Locumttenti / et Capitaneo Generalissimo, Regenti Chancellorium et doctoribus Ntrae. Regiae / Audientiae, Regenti Officium nrta. Generalis Gobernationis, et eius ordinario / Assesori Justitiae Aragonum et eius Locattenentibus, Baiulo Generali, Magistro / Rationali, Aduscato, et Procuratoribus fiscalibus, et Patrimonialibus Cal / metinis, Merinis Suprajunctanyis, Justitys, Juratis, Alguazeyis, Virgariys / et Portariys, Caeterisq. demun Uniuersis et singulis Officialibus et subditis / nostris maoiribus, et minoribus, indicto mo. Aragonum regno constitutis, et / Constituendis, dictorumq. Officialium Locatrnrntibus, seu officia ipsa regentibus / et subrogatis quouis modo pretibus. et futuris aduier sum ntra. Regiae indignationis / et irae, penaeq. florennorum aui Aragonum mille nostris Regis inferendore aerary / dicimus, praecipimus, et jubemus quattenus praeinsentam transactionem et concordiam / omniaq. et singula mea contenta a prima eius Linea vsq. ad ultimatum teneant firmi / ter, et obseruent, teneriq. et inviolabiter observari faciant ad vmquem per quoscumqe. / contrarium nullatenus tentaturi ratione aliqua siue causa, si dictus serenissimus / Princpes nobis morem perere Caeteri vero Officiales et subditi nostri praedicti gra / tiam nostram charam sabent ac praeter irae et indignationis nostrae meursum / paenam praeppositam cugiunt evitare. In cuius rei testimonium pritem fieri jussimo / nostro Regis Communi sigillo pendenti imunitam. Datty in Ciutitati nistra Caesaraugustae die vigesima mensis Novembris Anno a Natty. Domini / Millesimo Sexcentesimo Quadragesimo secundo, Regnorumq. Nostrum vigesmo. secundo.-Yo el Rey F. firmas ilegibles, y una diligencia: Confirma V. Magd. la concordia hecha entre el Lugar del Grado en este Reyno / de Aragon y la Ciudad de Caragoça sobre la vnion de aquel Lugar con esta Ciudad. Fd. P. Navarro. 
REVL-1976, núm. 190. ORDUÑA REBOLLO, ENRIQUE. CONCORDIA ENTRE EL LUGAR DEL GRADO...

REVL-1976, núm. 190. ORDUÑA REBOLLO, ENRIQUE. CONCORDIA ENTRE EL LUGAR DEL GRADO... 


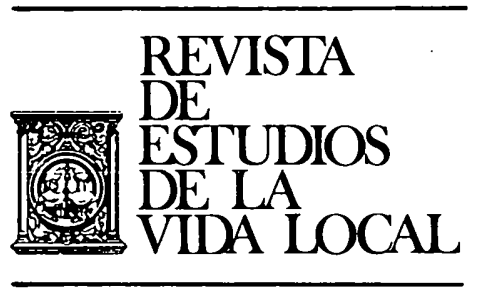

\section{ESTADISTICA}


REVL-1976, núm. 190. ORDUÑA REBOLLO, ENRIQUE. CONCORDIA ENTRE EL LUGAR DEL GRADO...

REVL-1976, núm. 190. ORDUÑA REBOLLO, ENRIQUE. CONCORDIA ENTRE EL LUGAR DEL GRADO... 